G. McHale and M.I. Newton, Frenkel's method and the dynamic wetting of planar heterogeneous surfaces, Colloids and Surfaces, A206 (2002) 193-201.

\title{
Frenkel's Method and the Dynamic Wetting of Heterogeneous Planar Surfaces
}

\author{
G. M⿳⺈冂䒑 Hale and M.I. Newton \\ Department of Chemistry and Physics, The Nottingham Trent University, \\ Clifton Lane, Nottingham NG11 8NS, UK.
}

\begin{abstract}
The relationship between the edge velocity, $v_{E}$, and the dynamic contact angle, $\theta$, for the spreading of a small spherical cap type droplet on chemically and geometrically heterogeneous surfaces is examined using Frenkel's method. In this method, the change in surface free energy is equated to the viscous dissipation caused by Poiseuille flow inside the spherical cap. To describe dynamic wetting of a surface that is heterogeneous due to small variations in the local surface geometry of the solid, we introduce a simple Wenzel type correction for the ratio of the actual to geometric surface areas, $r$. The rate of change of surface free energy is then $\left(2 \pi r_{0}\right) \gamma_{L_{I}}(\cos \theta-r I) v_{E}$ where $r_{O}$ is the drop base radius, $I=\left(\gamma_{S V^{-}}\right.$ $\left.\gamma_{S L}\right) / \gamma_{L V}$ and the $\gamma_{i j}$ 's are the interfacial tensions. For partial wetting, $I=\cos \theta_{e}$ where $\theta_{e}$ is the equilibrium contact angle and when the viscous dissipation vanishes, Wenzel's relationship linking the equilibrium contact angle on a rough surface to that on a smooth surface is obtained. Using dimensional arguments, we suggest that for a surface with weak geometric heterogeneity, the viscous dissipation is of the form $k \eta r_{o} v_{E}^{2} / \tan (\theta / 2)$ where $\eta$ is the viscosity and $k$ is a numerical factor. Balancing the rate of change of the surface free energy with the viscous dissipation gives the edge speed proportional to $\tan (\theta / 2)\left(r \cos \theta_{e}\right.$-cos$\left.\theta\right)$, which for small angles and smooth surfaces reduces to the Tanner-de Gennes Law $v_{E} \propto \theta\left(\theta^{2}-\theta_{e}{ }^{2}\right)$. The influence of incomplete penetration of the fluid into the surface structure is also examined. An analogous relationship based on a smooth, but chemically heterogeneous surface is derived. This is shown to give Cassie's equation for the equilibrium contact angle. For complete wetting, Frenkel's method predicts Tanner's law $\theta-t^{-3 / 10}$ when the surface is smooth and a modified Tanner's law tending towards $\theta \sim t^{-3 / 4}$ when the surface has a weak geometric heterogeneity.
\end{abstract}

Keywords: Tanner's Law, Contact Angle, Wetting, Spreading, Heterogeneity, Roughness, Dynamic Wetting, Topography.

• Corresponding author: Telephone: +44 (0)115 8483383; email: glen.mchale@ $@$ ntu.ac.uk 


\section{Introduction}

Surface roughness is known to alter the wetting of surfaces [1]. The measured macroscopic equilibrium contact angle depends on both the chemical nature of the surface and the local surface geometry [2]. Experimentally the influence of local geometry (topography) on wetting has been demonstrated with a range of materials including, rough waxy surfaces [3], glass-bead based surfaces [4], sol-gel derived oxide surfaces [5] and patterned silica surfaces [6]. Much of this work was motivated by the spectacular demonstration in 1996 of a superhydrophobic (super-non-wetting) surface with an equilibrium contact angle approaching $180^{\circ}$. The achievement of many super-hydrophobic surfaces is due to the combination of local geometry to provide a large geometric area for a relatively small projected area and a chemical treatment of the surface, often using self-assembled monolayers, to provide intrinsically non-wetting properties. However, local geometry used in this manner also predicts two other less well-studied effects: enhanced film formation (super-wetting) and modification of dynamic wetting. Whilst work is in progress on modifying equilibrium wetting by producing chemically patterned surfaces $[7,8]$ and on studying wetting dynamics on such surfaces [9], there appears to be relatively little consideration of the dynamic wetting of rough or geometrically patterned surfaces.

A significant achievement in the theoretical understanding of dynamic wetting for the complete spreading case, was the derivation, by Tanner [10], from the equations for viscous flow in two dimensions of a simple law relating the edge speed, $v_{\mathrm{E}}$, to the cube of the dynamic contact angle. De Gennes [11] later considered the case of partial wetting and derived a modified Tanner's law, valid for small angles, that gives $v_{\mathrm{E}} \propto \theta\left(\theta^{2}-\theta_{\mathrm{e}}^{2}\right)$ where $\theta_{\mathrm{e}}$ is the equilibrium contact angle. One of the simplest experiments is to follow the evolution in time of the contact angle as a small droplet of liquid spreads across a surface. When the droplet size is significantly less than the capillary length and the surface is smooth the droplet shape takes the form of a spherical cap. For this shape, Tanner's law can be explicitly solved in the small angle limit to give $\theta \times(t+C)^{-3 / 10}$ where $C$ is a constant. This time dependence of the dynamic contact angle is in excellent agreement with experimental data for all, but the longest times [12]. Tanner also demonstrated that his law was accurate up to $90^{\circ}$ and could be extended to the full angular range by replacing the edge angle by a suitable empirical function. A good agreement with experimental data was obtained for a range of angles up to $165^{\circ}$ [13]. In all these cases, the surfaces considered were smooth and chemically homogeneous and the emphasis was either on complete wetting with $\theta_{\mathrm{e}}=0^{\circ}$ or partial wetting, but with a small value of the equilibrium contact angle. 
In 1945, Frenkel [14] outlined a method for obtaining the equilibrium contact angle of a spherical cap type droplet based on the change in surface free energy as the drop evolves. This method was later extended to examine the coalescence of spherical drops by including a viscous dissipation term modelled on a rod under uniaxial compression [15]. McHale et al [16] applied the same ideas to the dynamic spreading of a small spherical cap type droplet on a smooth and chemically homogeneous surface by using a viscous dissipation based on Poiseuille flow within a cone inscribed within the droplet. This approach enabled an edge velocity-contact angle relationship to be obtained for partial wetting and for a wide range of dynamic contact angle. It was shown that the angular function obtained could accurately describe the experimental data and so replace the empirical function used by Tanner. In the small angle limit, the formula reduces to the Tanner - de Gennes law for the edge velocity.

In this article, we first review simple models describing equilibrium contact angles on heterogeneous surfaces, but with an emphasis on changes in surface free energy. Frenkel's method is then developed and applied to the case of heterogeneous surfaces. Both surfaces with chemical heterogeneity and surfaces which are chemically homogeneous, but which are rough or have local geometric (topographic) patterns are considered. Modifications of the equilibrium equations of Cassie and Wenzel to the dynamic case are considered. Equations analogous to the Tanner-de Gennes law are derived using both a simple dimensional argument for the viscous dissipation and a model of Poiseiulle flow within a cone inscribed within the spherical cap shaped droplet. The possible influence of incomplete liquid penetration into surface features is also considered. For the case of chemically homogeneous surfaces that have a characteristic roughness, possibly due to geometric patterns, the small angle limit for the dynamic contact angle is derived. For a smooth surface showing complete wetting and a dynamic contact angle $\theta \propto\left(t+C_{1}\right)^{-3 / 10}$, it is suggested that the rough surface may have a modified power law tending towards $\theta \times\left(t+C_{2}\right)^{-3 / 4}$. It is also argued that the dynamic contact angle may enable surfaces that are super-wetting, in the sense of having a vanishing equilibrium contact angle due to surface roughness, but which differ in their equilibrium contact angles on the smooth surfaces to be distinguished from each other.

\section{Review of Equilibrium Contact Angles}

In this section, standard approaches to equilibrium wetting are considered with an emphasis on changes in the surface free energy. This brief review underpins the application in section 3 of Frenkel's method to dynamic wetting on heterogeneous surfaces. 


\subsection{Smooth heterogeneous surfaces}

On smooth and chemically homogeneous planar surfaces, two approaches to the equilibrium contact angle, $\theta_{\mathrm{e}}$, exist, i) force balance, and ii) minimum surface free energy. In the force view (Fig. 1a), the interfacial tensions, $\gamma_{i j}$, are regarded as forces per unit length and a horizontal force balance at the contact line is required: $\gamma_{\mathrm{L}}+\gamma_{\mathrm{LV}} \cos \theta_{\mathrm{e}}=\gamma_{\mathrm{SV}}$. In the energy view, the interfacial tensions, $\gamma_{i j}$, are regarded as energies per unit area, and the surface free energy change, $\Delta F$, due to a displacement, $\Delta A$, of the contact line is considered (Fig. 1b). This energy change of, $\Delta F=\left(\gamma_{\mathrm{L}}-\gamma_{S \mathrm{~V}}\right) \Delta A+\gamma_{\mathrm{V}} \cos \theta_{\mathrm{e}} \Delta A$ must vanish when the contact angle is at its equilibrium value. Either approach gives rise to Young's equation,

$$
\cos \theta_{e}=\left(\gamma_{S V}-\gamma_{S L}\right) / \gamma_{L V}
$$

On a heterogeneous surface problems can arise with the force view because the surface may be continuous, but non-differentiable so preventing a simple resolving of forces. In contrast, the energy view provides a simple approach to surfaces that are patterned or rough, irrespective of whether the patterning is chemical or geometric.

\subsection{Chemically heterogeneous surfaces}

Consider a surface which is smooth, but which is a composite of microscopic patches with two types of chemical properties characterised by different solid-liquid and solid-vapor interfacial tensions, $\gamma_{i j}{ }^{1}$ and $\gamma_{i j}{ }^{2}$. The overall surface can be characterised by a factor, $f=s_{1} /\left(s_{1}+s_{2}\right)$, where $s_{i}$ is the typical area of a patch of chemical properties of type $i$. Displacing the liquid-vapor interface by a small area on the solid surface of $\Delta A$, as shown in fig. $2 \mathrm{a}$, gives a surface free energy change of,

$$
\Delta F=\left(\gamma_{S L}^{1}-\gamma_{S V}^{1}\right) f \Delta A+\left(\gamma_{S L}^{2}-\gamma_{S V}^{2}\right)(1-f) \Delta A+\gamma_{L V} \cos \theta \Delta A
$$

assuming changes in contact angle are second order. Requiring this change in surface free energy to vanish at equilibrium gives Cassie's equation,

$$
\cos \theta_{e}=f \cos \theta_{1}+(1-f) \cos \theta_{2}
$$

where $\theta_{1}$ are the equilibrium contact angles given by eq. (1) for each of the two types of surface forming the composite surface. 


\subsection{Geometrically heterogeneous surfaces}

The geometric case, involving a surface that is chemically homogeneous, but which has local roughness or geometric structure can also be considered within the surface free energy approach. Consider characterising such a surface by a roughness factor, $r=\Delta A_{\text {True }} / \Delta A$, where $\triangle A$ is the horizontal projection of the surface microscopic area. In the sense used in this work, the factor $r$ does not only represent surface roughness, but may alternatively represent the enhanced microscopic surface area resulting from a deliberate patterning of the surface $[6,17]$. In this latter case, the shape of the pattern and the aspect ratio in the fabrication of the surface can be directly related to the "roughness" factor $r$. Considering a small displacement, $\Delta A$, of the liquid-vapor interface, as shown in fig. $2 \mathrm{~b}$, gives a surface free energy change of,

$$
\Delta F=\left(\gamma_{S L}-\gamma_{S V}\right) r \Delta A+\gamma_{L V} \cos \theta \Delta A
$$

The geometric influence arises only in the solid-liquid and solid-vapor interfacial areas, provided the contact angle change is second order. Requiring the change in surface free energy to vanish to first order results in Wenzel's equation,

$$
\cos \theta_{e}=r\left(\gamma_{S V}-\gamma_{S L}\right) / \gamma_{L V}=r \cos \theta_{e}^{s}
$$

where $\theta_{e}$ and $\theta_{e}^{s}$ are the contact angles on the rough and smooth surfaces, respectively. The derivations of Cassie's equation and Wenzel's equation are simple within the framework of minimising surface free energy changes. However, they involve significant assumptions including, i) the change in contact angle is second order, ii) the axial symmetry of the drop is maintained, and iii) the displacement of the liquid-vapor interface proceeds with the solid surface maintaining intimate contact with the liquid at all locations. Experimentally, some of these assumptions are known to not always hold. For example, drops on patterned surfaces when viewed from above may not show a circular shape, but may take on the symmetry of the shapes used to create the pattern. Hexagonal patterns of surface protrusions can give rise to pinning and coherent motion of the contact line, such that the equilibrium drop shape is hexagonal when viewed from above [8].

Wenzel's equation is of particular interest because it predicts that equilibrium wetting behaviour induced by surface chemistry can be emphasised by geometry. This is illustrated by the solid line in fig. 3 , which illustrates the effect of eq. (5) for a roughness factor $r=5$. 
Three regions can be observed, i) super-hydrophobicity ("planar roll-up"), ii) superhydrophilicity ("film formation"), and iii) the region in-between representing contact angle "amplification". Wenzel's equation predicts that for $\theta_{\mathrm{e}}^{\mathrm{s}}>90^{\circ}$ the effect of roughness is to increase the contact angle towards $180^{\circ}$ and for $\theta_{\mathrm{e}}^{\mathrm{s}}<90^{\circ}$ the effect is to decrease it towards $0^{\circ}$. Experimentally, it is found that the threshold of $90^{\circ}$ for the smooth surface contact angle that determines which direction surface roughness modifies the contact angle can be offset to a lower value. Quéré et al [6] (see also Adamson [2]) explained the offset by considering a breakdown in the assumption that intimate contact is maintained by the liquid with the solid. Consider incomplete liquid penetration into a rough surface so that vapor exists trapped in small pockets between the solid and the liquid, as shown in fig. 4. To model this system two parameters, $f$ and $g$ are introduced with $f$ representing the fraction of the rough solid surface covered by the liquid and $g$ the projected area of trapped vapor not in contact with the liquid. It is to be expected that these parameters depend on the contact angle and that they are interrelated such that when $f=1$ then $g=0$. In the approach of Quére et al, $f$ is approximated to a constant independent of the contact angle and $g$ is taken as $f$-1. The displacement of the liquid-vapor interface, shown in fig. 4 , gives a surface free energy change of,

$$
\Delta F=\left(\gamma_{S L}-\gamma_{S V}\right) f r \Delta A+\gamma_{L V} g \Delta A+\gamma_{L V} \cos \theta \Delta A
$$

and the modified Wenzel's equation is then,

$$
\cos \theta_{e}=r f \cos \theta_{e}^{s}-g
$$

In a sense, the model considered by Quéré et al is one of a composite surface involving both geometric structure through the $r$ factor and chemical structure through the presence of both a solid and trapped vapor at the interface with the solid. The effect of trapped vapor in the manner suggested by eq. (7) is illustrated in fig. 3 by the dotted line using parameters $f=0.7$ and $g=0.3$. Clearly the threshold value of the smooth surface contact angle that determines whether surface roughness generates a higher or lower contact angle can be reduced below $90^{\circ}$ by the effect of vapor trapping. In addition, vapor trapping alters the slope of the curve in the contact angle amplification region and the values of $\theta_{\mathrm{e}}{ }^{\mathrm{s}}$ for which saturation occurs.

Whilst Wenzel's equation has been known for many years, it is only relatively recently that it has been used to design surfaces that amplify wetting behaviour [3]. Most of the recent work 
focuses on the creation of super-hydrophobic or super non-wetting surfaces and the relevance of Wenzel's equation to both the opposite super-hydrophilic/wetting case and to dynamic wetting has been relatively neglected. Indeed, we expect that dynamic wetting, the rate at which the contact angle approaches its equilibrium value, will be relevant to both super-nonwetting surfaces and super-wetting surfaces. In particular, it is possible to envisage that dynamics could allow two surfaces with $\theta_{e}=0^{\circ}$, but which have different values of $\theta_{\mathrm{e}}^{\mathrm{s}}$, to be distinguished.

\section{Frenkel's Method for Dynamic Wetting on Heterogeneous Surfaces}

Popular methods of modelling dynamic wetting on homogeneous planar surfaces include, hydrodynamic theory [11,12] and molecular-kinetic theory [18,19]. In Frenkel's method [1416] the rate of surface free energy change of a given shape, in this case a constant volume spherical cap, are deduced and these are equated to a rate of viscous dissipation obtained from a hydrodynamic approach. The advantages of the method are, i) on smooth chemically homogeneous surfaces the correct limit for small $\theta_{\mathrm{e}}$ is obtained, ii) an edge speed-wide angle relation can be derived, and iii) the partial wetting $\left(\theta_{\mathrm{e}} \neq 0^{\circ}\right)$ case can be modelled.

\subsection{Surface free energy changes}

The analogous case to Wenzel's equation of a rough surface having intimate contact between liquid and solid will be considered in detail and results for the composite case and the chemically heterogeneous case simply stated. Consider the spherical cap geometry shown in fig. 5. The spherical radius $R$, contact radius $r_{\mathrm{o}}$, cap height $h_{\mathrm{o}}$, and volume $V$, are related by the equations,

$$
R=\left(\frac{3 V}{\pi \beta}\right)^{1 / 3} \quad r_{o}=R \sin \theta \quad h_{o}=R(1-\cos \theta)
$$

where $\beta$ is a function of the contact angle and is defined as,

$$
\beta=2-3 \cos \theta+\cos ^{3} \theta=(1-\cos \theta)^{2}(2+\cos \theta)
$$

The total surface free energy $F$, is due to the three surfaces separating the liquid, vapor and solid,

$$
F=F_{o}+\gamma_{L V} A_{L V}+\left(\gamma_{S L}-\gamma_{S V}\right) A_{S L}
$$


where $F_{\mathrm{o}}$ is a constant. From the spherical cap geometry, the liquid-vapor and the solid-liquid surface areas are,

$$
A_{S L}=r \pi r_{o}^{2} \quad A_{L V}=2 \pi R^{2}(1-\cos \theta)
$$

where the factor of $r$ is the geometric "roughness" factor defined as $r=\Delta A_{\text {True }} / \Delta A$, and $\Delta A$ is the horizontal projection of the surface microscopic area.

Differentiating eq. (10), and performing some algebraic manipulation gives the rate of dissipation of surface free energy as,

$$
\frac{d F}{d t}=2 \pi \gamma_{L V}(\cos \theta-r I) r_{o} v_{E}
$$

where $I$ has been defined as,

$$
I=\frac{\gamma_{S V}-\gamma_{S L}}{\gamma_{L V}}
$$

and the edge speed is given by,

$$
v_{E}=\frac{-(3 V / \pi)^{1 / 3} d \theta / d t}{(1-\cos \theta)^{2 / 3}(2+\cos \theta)^{4 / 3}}
$$

If the calculation is modified to allow for trapping of vapor using the two constant factors, $f$ and $g$, defined in section 2.3 , eq. (12) is modified to,

$$
\frac{d F}{d t}=2 \pi r_{o} \gamma_{L V}[\cos \theta-f r I+g] v_{E}
$$

Similar algebraic manipulations can be performed for the smooth, but chemically heterogeneous surface and the result is then,

$$
\frac{d F}{d t}=2 \pi r_{o} \gamma_{L V}\left[\cos \theta-f I_{1}-(1-f) I_{2}\right] v_{E}
$$


One immediate consequence of eq. (12) and eq. (16) is that if the rate of change of surface free energy vanishes, Wenzel's and Cassie's equations are obtained and define the equilibrium contact angles.

\subsection{Form of dissipation}

In cylindrical co-ordinates, centred on the base of a spherical cap, the rate of dissipation of energy in the fluid is given by [16],

$$
\frac{d E_{d}}{d t}=\eta \int_{\text {liquid }} \phi_{v} d V
$$

where $\eta$ is the viscosity and $\phi_{v}$ is given in terms of the radial, $v_{r}$, and axial, $v_{z}$, velocity components by,

$$
\phi_{v}=2\left[\left(\frac{\partial v_{r}}{\partial r}\right)^{2}+\left(\frac{v_{r}}{r}\right)^{2}+\left(\frac{\partial v_{z}}{\partial z}\right)^{2}\right]+\left(\frac{\partial v_{r}}{\partial z}+\frac{\partial v_{z}}{\partial r}\right)^{2}
$$

A detailed knowledge of the velocity field is needed to evaluate eq. (18) and so obtain a viscous dissipation from eq. (17). However, a simple estimate can be found by performing an analysis of the characteristic dimensions of the terms in eq. (18). Taking the fluid to be incompressible and using the continuity equation shows that there are three types of terms contributing to the dissipation of energy,

$$
\frac{v_{E}^{2} r_{o}^{2}}{h_{o}} \quad v_{E}^{2} h_{o} \quad \frac{v_{E}^{2} h_{o}^{3}}{r_{o}^{2}}
$$

The first term, which is dominant, arises from the variation of the radial velocity with the spherical cap height. Thus, noting that the ratio of cap height to contact radius is given by $\tan (\theta / 2)$, the viscous dissipation is anticipated to be of the form,

$$
\frac{d E_{d}}{d t}=\frac{k \eta v_{E}^{2} r_{o}}{\tan (\theta / 2)}
$$

where $k$ is a constant. 
Several routes exist to determining a more precise form for the viscous dissipation. The most popular is to consider Poiseuille flow within a wedge shaped edge of the droplet $[11,12]$. The velocity field is then,

$$
v_{r}(z)=v_{E}\left[-\left(\frac{z}{h(r)}\right)^{2}+2\left(\frac{z}{h(r)}\right)\right]
$$

Another approach, considered by Strella [15] for the coalescence of spherical drops, was to consider a uniaxially compressed rod model for the energy dissipation. Denesuk et al [20] built on this idea by considering a uniaxially contracting cylinder of fluid. It is interesting to note that a similar idea has recently been used in the recent work of de Ruijters et al [21], who derived a viscous dissipation occurring from the velocity field in the core of the spreading drop from modelling the cap as a spreading cylindrical disk. They also included an additional term to represent dissipation due to frictional effects in the vicinity of the contact line. An extension of the idea of a uniaxially contracting cylinder of fluid suggested by Denesuk et al [20], was proposed by McHale et al [16] who suggested using a Poiseuille flow within a cone inscribed within the spherical cap (fig. 6). The rate of energy dissipation then evaluates to,

$$
\frac{d E_{d}}{d t}=\frac{8 \pi \eta v_{E}^{2} r_{o} J_{W}}{3 \tan (\theta / 2)}
$$

where $J_{\mathrm{W}}$ is given by,

$$
J_{W}=\varepsilon-\log _{e} \varepsilon-1
$$

and arises from a truncated integral that regularises a singularity. The parameter $\varepsilon$ is a cut-off in the evaluation of the integral and can be physically interpreted as due to some finite height at which the macroscopic cap passes into a film [16]. The result (eq. (22)) from the cone model provides a definition for the constant factor, $k$, arising from the dimensional analysis. A significant difference between the wedge approximation and the cone approximation, is the occurrence of $a \tan (\theta / 2)$ in the denominator of eq. (22) rather than a $\tan \theta$. The half-angle is significant in enabling the subsequent version of Tanner's law to be accurately fitted to the wide angle data from experiment. 


\subsection{Edge speed-contact angle relation for heterogeneous surfaces}

Balancing the dissipation for the cone, eq. (22), with the rate of change of the surface free energy (eq. (12), (15) or (16)) gives a relationship between the edge speed and the dynamic contact angle. For the case of a chemically heterogeneous surface the appropriate rate of energy change is given by eq. (16) and the resulting relationship is,

$$
v_{E}=\left(\frac{2 \pi \gamma_{L V}}{k \eta}\right) \tan (\theta / 2)\left[f I_{1}+(1-f) I_{2}-\cos \theta\right]
$$

For the composite geometric surface, eq (15) is used for the rate of energy change and this gives the relationship,

$$
v_{E}=\left(\frac{2 \pi \gamma_{L V}}{k \eta}\right) \tan (\theta / 2)[f r I-g-\cos \theta]
$$

The effect of a frictional term in the dissipation [21] could be incorporated into these equations in a straightforward manner and would lead to the $\tan (\theta / 2)$ prefactor being replaced by a factor of the form $[a+\tan (\theta / 2)]$ where $a$ would be a constant independent of the dynamic contact angle.

A simple limiting case for the chemically homogeneous geometric surface can be obtained from eq. (25) using the small angle limit,

$$
v_{E}=\frac{\pi \gamma_{L V}}{k \eta} \theta\left(f r \cos \theta_{e}^{s}-g-\cos \theta\right) \approx \frac{\pi \gamma_{L V}}{k \eta} \theta\left((f r-g-1)+\left(\theta^{2}-\theta_{e}^{s^{2}}\right) / 2\right)
$$

This is the Tanner-de Gennes equation ( $f r=1$ and $g=0$ ) modified by the Wenzel roughness factor.

\subsection{Dynamic contact angle - complete wetting on a geometrically heterogeneous surface}

In experimental studies of spreading, the dynamic contact angle is often measured as function of time. For complete wetting where $\theta_{\mathrm{e}}^{\mathrm{s}}=0^{\circ}$, a simple power law, Tanner's law $\theta \sim t^{-3 / 10}$, has 
been well verified for oils on surfaces such as silicon [12], although some discrepancies have been noted particularly at long times [22]. Since we are interested in how the equivalent of Wenzel's ideas on surface roughness or geometric patterning of the surface modifies dynamic wetting, a simple power law for the complete wetting case may be of value. For a spherical cap the edge speed, $v_{\mathrm{E}}$, is related to the contact angle and volume, $V$, by eq. (14) and in the small angle approximation this gives,

$$
v_{E}=\frac{-(4 V / 27 \pi)^{1 / 3} d \theta / d t}{\theta^{4 / 3}}
$$

For a smooth, high energy surface having $\theta_{\mathrm{e}}^{\mathrm{s}}=0^{\circ}$, eq. (26) and eq. (27) give,

$$
\frac{d \theta}{d t} \propto-\theta^{7 / 3}\left[2(f r-g-1)+\theta^{2}\right]
$$

When $f r=1$ and $g=0$, or when the surface roughness can be ignored, Tanner's law is recovered, but when the surface is rough or geometric patterning dominates the dynamics a modified power law is obtained,

$$
\theta \approx \frac{A(f r-g-1)}{(t+C)^{3 / 4}} \sim \frac{1}{t^{3 / 4}}
$$

where $A$ and $C$ are constants. The model therefore predicts that a power law with an exponent intermediate between $-3 / 10$ and $-3 / 4$ may be observed.

\subsection{Validity of the approach}

Frenkel's method is a simple approach based on surface free energy changes and viscous dissipation. It has the advantage of giving the accepted limits of an edge speed proportional to the cube of the dynamic contact angle and of a contact angle dependence of $t^{-3 / 10}$ for dynamics on smooth surfaces. The method, as applied within this work, provides an approach to the dynamic contact angle on chemically and geometrically heterogeneous surfaces which is consistent with Wenzel's and Cassie's equations for the equilibrium contact angle. However, the approach is limited in assuming a spherical cap droplet with its inherent axial symmetry which is unlikely to be the case when surfaces are strongly heterogeneous due to patterns that have an imposed symmetry [8]. Furthermore, the form of the viscous dissipation 
has been assumed to be the same on the heterogeneous surface as on the smooth surface and it is far from clear whether this will be valid. It is therefore likely that the results of the Frenkel's method approach can at best represent an average effect and are likely to be limited to the case of a weak influence of heterogeneity. The method does not address the issue of contact line pinning. In addition, for the geometric heterogeneous case, i.e. due to surface topography, the approach does not include a rigorous relationship between the $f$ and $g$ parameters and the contact angle on the smooth surface. The influence of incomplete penetration of fluid into surface features may therefore be a problem. Despite these limitations, the method does suggest that combining surface geometry with surface chemical treatments can provide more than the super-non-wetting/hydrophobic surfaces that have been of recent experimental interest. The super-wetting/hydrophilic case, which Wenzel's equation predicts will enhance film formation, should also be of interest. Our work predicts that the dynamics of contact angles on such surfaces may be significantly different to the case of smooth, chemically homogeneous surfaces. Whether eq. (29) turns out to be an accurate description cannot be determined from existing data, but it should provide additional motivation for new experimental studies.

\section{Conclusions}

The relationship between heterogeneous surfaces and the dynamic contact angle has been considered. Frenkel's approach based on surface free energy changes and viscous dissipation has been applied to both chemically heterogeneous surfaces and rough/geometrically structured surfaces. It has been shown that this formulation contains both Cassie's and Wenzel's equations as limiting cases. Edge speed-contact angle relations have been derived for both types of heterogeneous surfaces thus giving a generalised Tanner-de Gennes law. This law describes both a wide range of dynamic contact angles and the complete and partial wetting cases. For a geometrically structured or rough surface the effect of vapor trapping has been considered. The time dependence of the dynamic contact angle for superwetting/hydrophilic surfaces has been examined and a simple power law derived.

\section{Acknowledgement}

The financial support of the UK Engineering and Physical Sciences Research Council (EPSRC) under grant GR/02184 is gratefully acknowledged. 


\section{References}

1. R.N. Wenzel, Ind. Eng. Chem., 28988 (1936).

2. A.W. Adamson and A.P. Gast, Physical Chemistry of Surfaces, $6^{\text {th }}$ ed. J. Wiley \& Sons (1997).

3. T. Onda, S. Shibuichi, N. Satoh and K. Tsujii, Langmuir, 122125 (1996).

4. M. Fuiji, H. Fukimori, T. Takei, T. Wanabe and M. Chikazawa, J. Phys. Chem. B, 102 $1048(1998)$

5. K. Tadanaga, N. Katata and T. Minami, J. Am. Ceram Soc., $\underline{80} 1040$ (1997).

6. D. Quéré and D. Richard, Europhys Lett., $\underline{48} 286$ (1999).

7. M. Voué and J. De Coninck, Oil \& Gas Sci. Technol - Rev IFP, 5697 (2000).

8. T. Cubaud, M. Fermigier and P. Jenffer, Oil \& Gas Sci. Technol - Rev IFP, $\underline{56} 23$ (2000).

9. S. Semal, M. Voué, M.J. De Ruijter, J. Dehuit and G. De Coninck, J. Phys. Chem. B., 103 4854 (1999).

10. L.H. Tanner, J.Phys. D: Appl. Phys., 121473 (1979).

11. P. G. De Gennes, Rev. Mod. Phys., 57827 (1985).

12. L. Léger and J.F. Joanny, Rep. Prog. Phys., 55431 (1992).

13. L.H. Tanner, J.Phys. D: Appl. Phys., 19751 (1986).

14. J. Frenkel, J. Phys. (USSR), 9385 (1945).

15. S. Strella, J. Appl. Phys., 414242 (1970).

16. G. McHale, S.M. Rowan and M.I. Newton, J.Phys. D: Appl. Phys., 272619 (1994).

17. A. Kawai and H. Nagata, J. J. Appl. Phys., $\underline{33}$ L1283 (1994).

18. T.D. Blake and J.M. Haynes, J. Colloid Interf. Sci., 30421 (1969).

19. T.D. Blake in Wettability, J.C. Berg (Ed) Marcel Dekker New York pp251-310 (1993).

20. M. Denesuk, J.P. Cronin, B.J.J. Zelinski, N.J. Keidl and D.R. Uhlmann, Phys. Chem. Glasses, $\underline{34} 203$ (1993).

21. M.J. De Ruijter, J. De Coninck and G. Oshanin, Langmuir 152209 (1999).

22. P. Silberzan and L. Léger, Phys. Rev. Lett., 66185 (1991). 


\section{Figure Captions}

Figure 1. Equilibrium contact angle on smooth, chemically homogeneous surfaces using, (a) force balance, and (b) minimum surface free energy.

Figure 2. Equilibrium contact angle from minimum surface free energy for, (a) chemically heterogeneous surface, and (b) geometric/rough surface.

Figure 3. Effect of Wenzel's equation for a "roughness" $r=5$ (solid line). The dotted line shows the model of vapor trapping with $f=0.7$ and $g=0.3$. (see comment on diagram about error in original paper).

Figure 4. Minimum surface free energy from a liquid-vapor interface displacement when vapor trapping occurs.

Figure 5. Spherical cap geometry for a spreading droplet.

Figure 6. Viscous dissipation modelled by radial flow within a cone inscribed within the spherical cap. A cut-off occurs at a characteristic height $z_{\mathrm{o}}$ a distance of $\varepsilon$ from the edge of the droplet. 

(a)

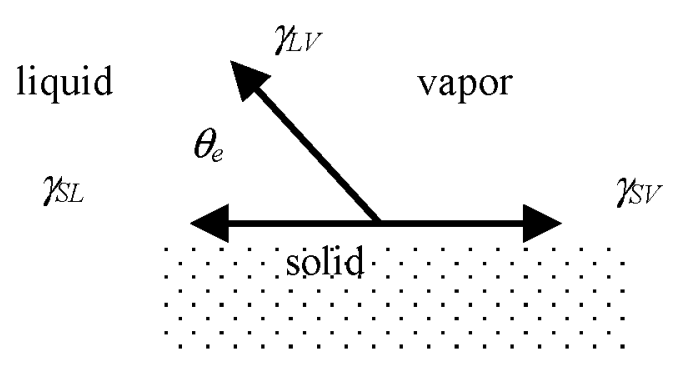

(b)

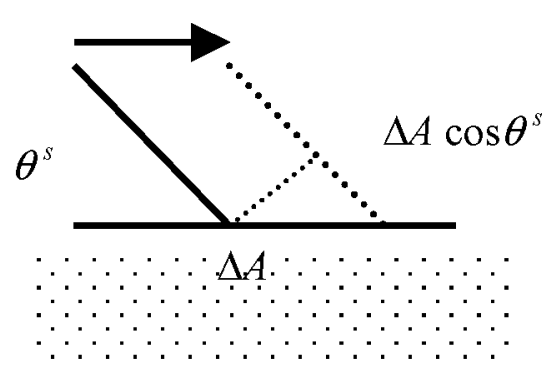

Figure 1 McHale and Newton, Frenkel's method and the dynamic wetting of heterogeneous planar surfaces. 
(a)

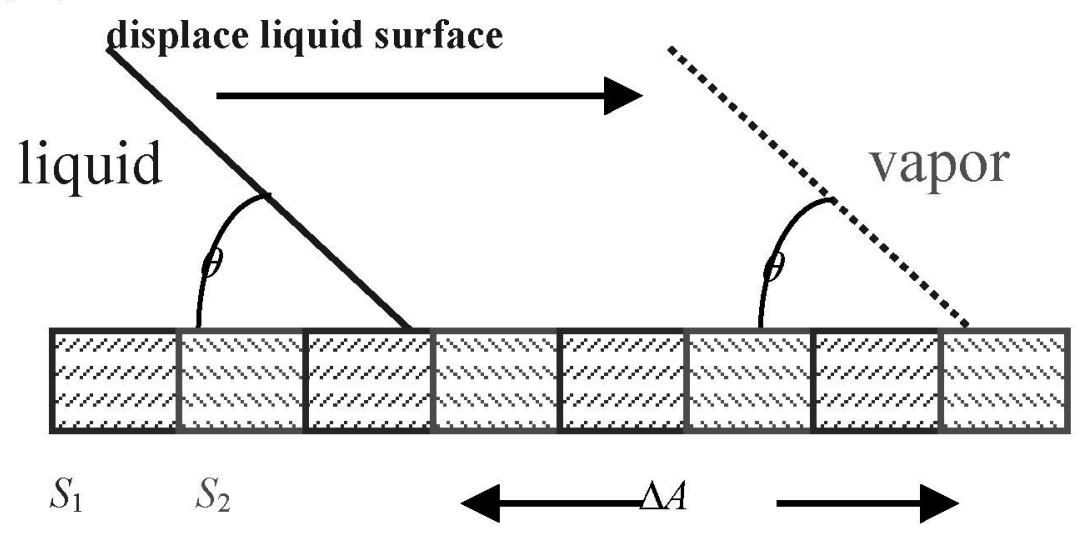

(b)

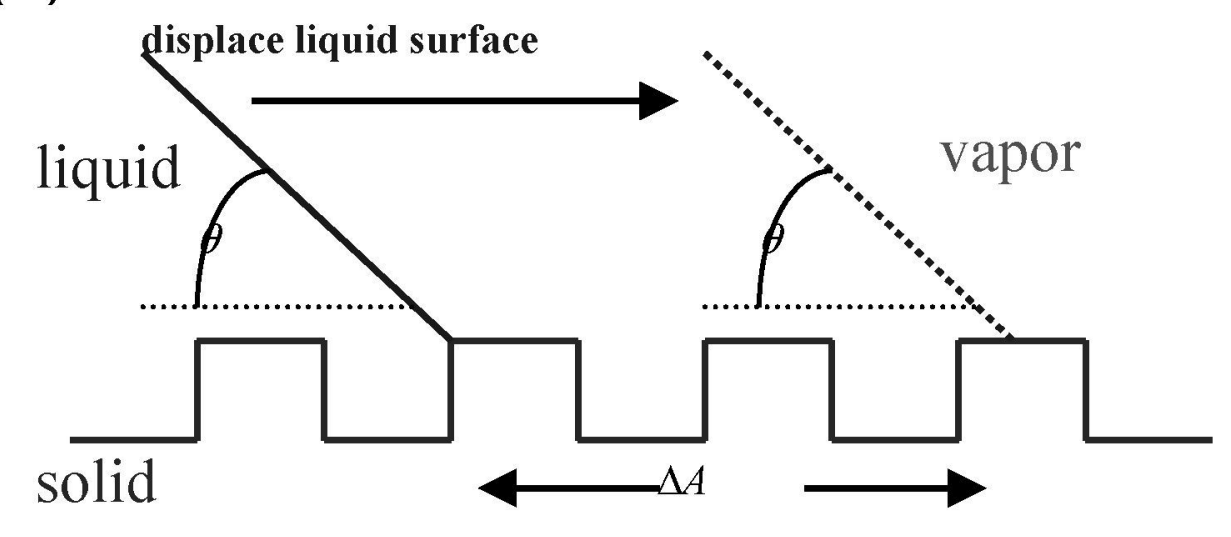

Figure 2 McHale and Newton, Frenkel's method and the dynamic wetting of heterogeneous planar surfaces. 


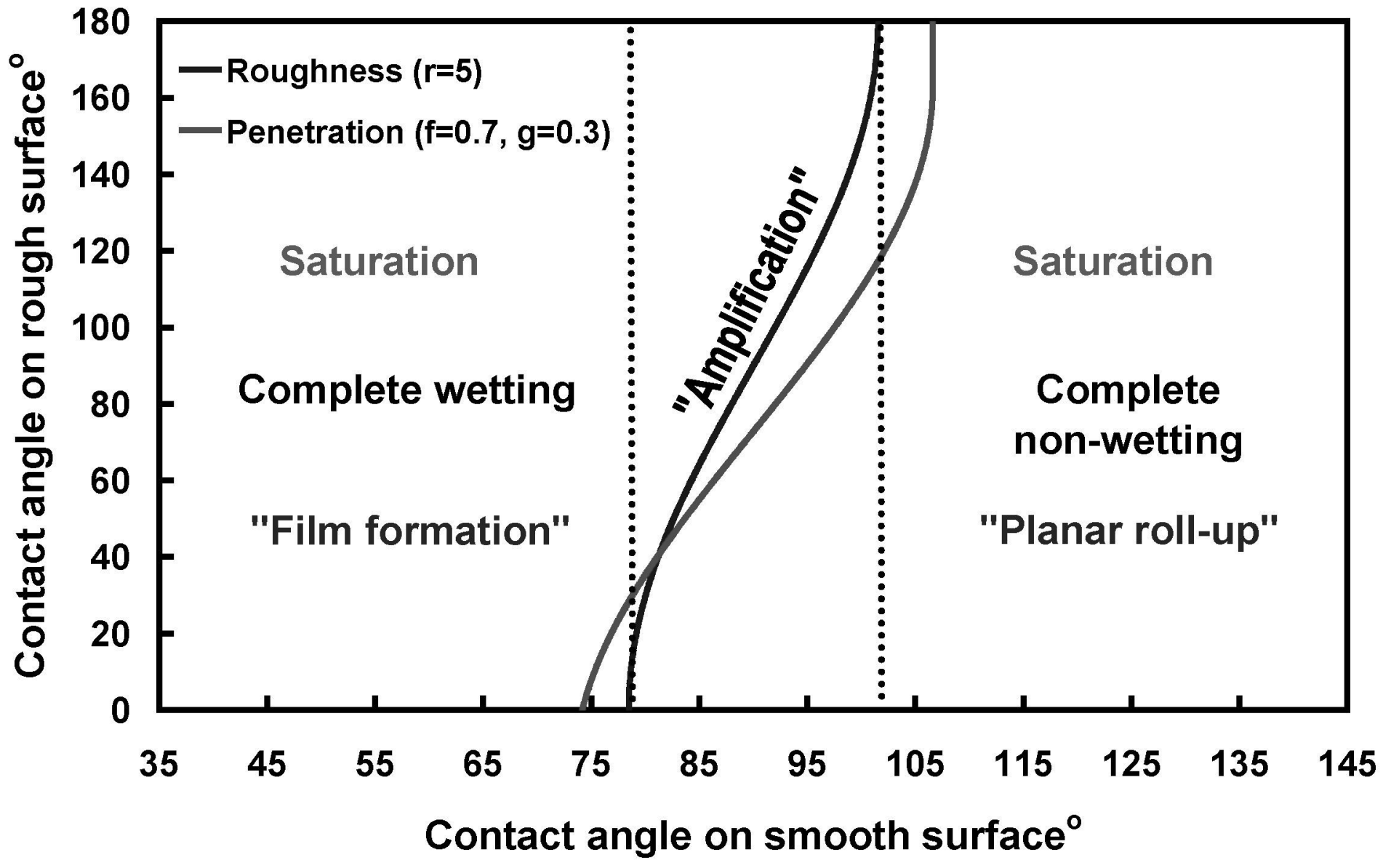

Figure 3 McHale and Newton, Frenkel's method and the dynamic wetting of heterogeneous planar surfaces. This original published figure was in error due to a mistake in the spreadsheet calculation. This error does not alter the equations or conclusions of the paper. 


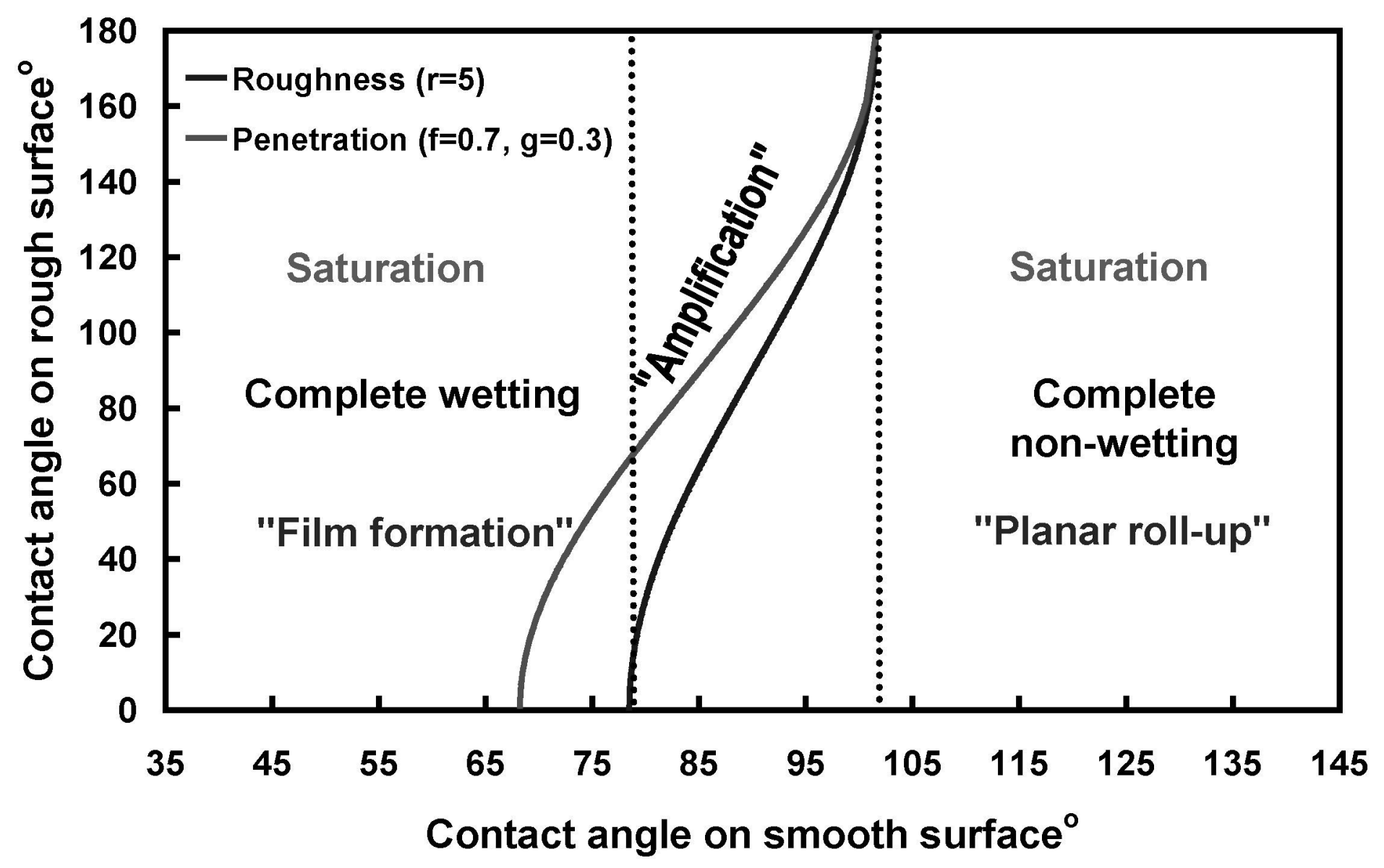

Figure 3 McHale and Newton, Frenkel's method and the dynamic wetting of heterogeneous planar surfaces. This is the corrected figure 3 


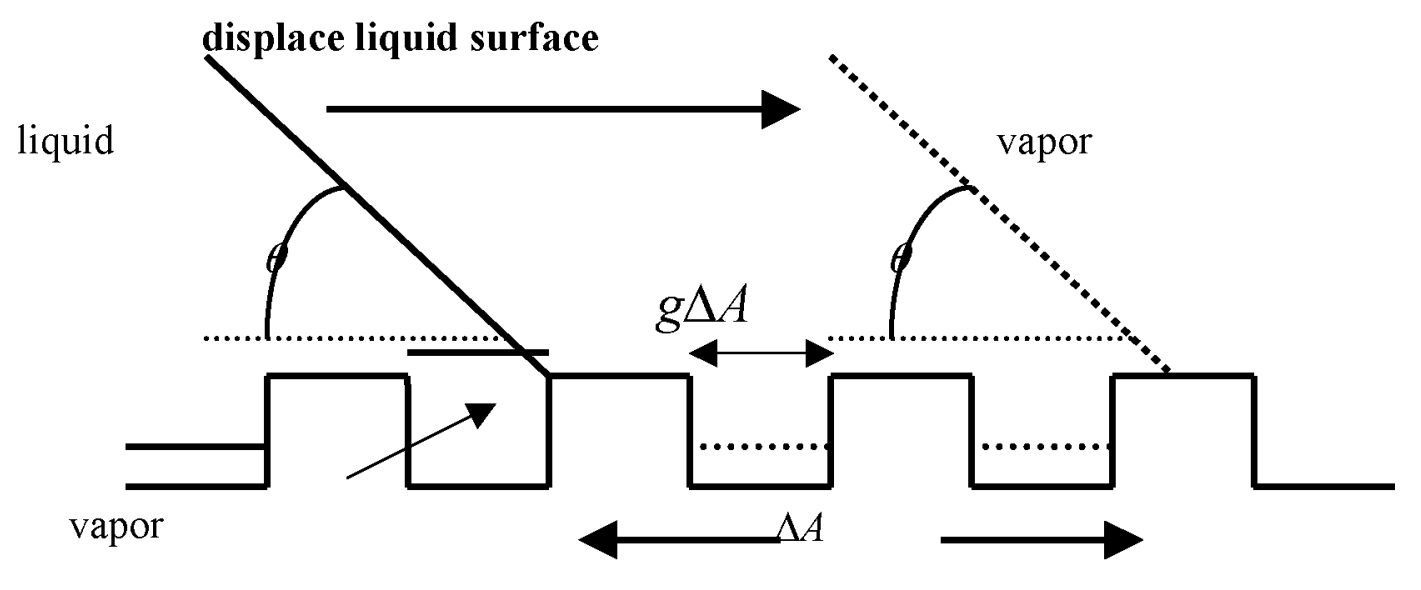

Figure 4 McHale and Newton, Frenkel's method and the dynamic wetting of heterogeneous planar surfaces. 


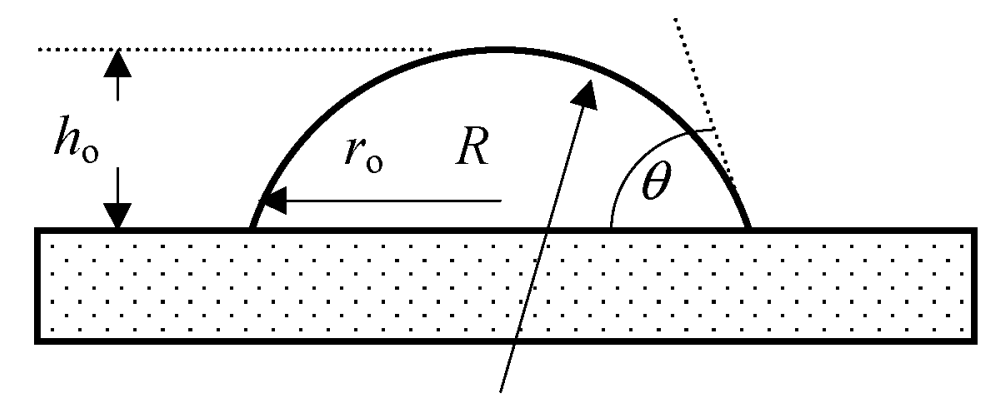

Figure 5 McHale and Newton, Frenkel's method and the dynamic wetting of heterogeneous planar surfaces. 


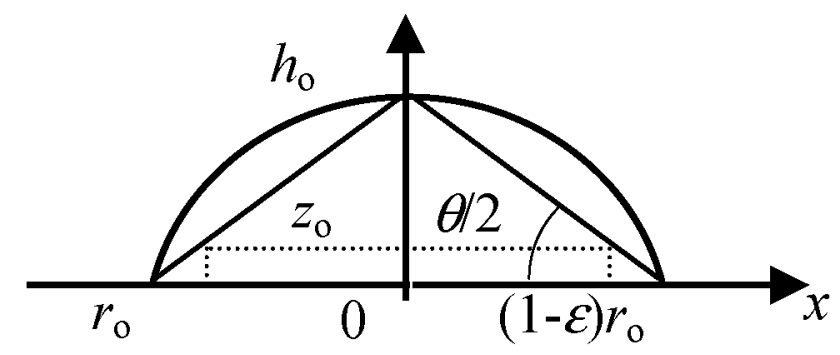

Figure 6 McHale and Newton, Frenkel's method and the dynamic wetting of heterogeneous planar surfaces. 